\title{
The Importance of Radio Allergosorbent Testing Early in the Management of Atopic Dermatitis in Children referred to Secondary Care
}

\author{
Mohammed Al-Abadie*1, Mohammed Al-Rubaye ${ }^{1}$, Faris Oumeish ${ }^{1}$ and Dina Al-Abadie ${ }^{2}$ \\ ${ }^{1}$ Royal Wolverhampton NHS Trust, United Kingdom \\ ${ }^{2}$ Department of Health Promotion and Public Health, University of West London, United Kingdom
}

*Corresponding author: Mohammed Al-Abadie, Royal Wolverhampton NHS Trust, Wolverhampton, United Kingdom

\section{ARTICLE INFO}

Received: 幽 May 10, 2019

Published: 慧 May 20, 2019

Citation: Mohammed Al-Abadie, Mohammed Al-Rubaye, Faris Oumeish, Dina Al-Abadie. The Importance of Radio Allergosorbent Testing Early in the Management of Atopic Dermatitis in Children referred to Secondary Care. Biomed J Sci \& Tech Res 18(1)-2019. BJSTR. MS.ID.003108.

Keywords: RAST testing; Atopic dermatitis; Allergy; IgE

\section{ABSTRACT}

Background: Atopic dermatitis patients are usually diagnosed and managed by Primary Care Practice. However, those who fail treatment are usually sent to Specialist Secondary Care Dermatologist for further assessment and management. RAST testing is one of the options used by Dermatologists in their investigations.

Aim: The aim of this study is to evaluate the relevance of radio allegro sorbent testing (RAST) in the management of paediatric patients with atopic dermatitis.

Method: Standard RAST testing was requested for all patients referred to secondary care with AD. Allergens tested included: dairy products, house dust mite, grass, nuts, seafood, cereals, dog dander and cat epithelium.

Results: 133 patients suffering from eczema referred to secondary care were tested. RAST test showed that $91.7 \%$ of children having high/abnormal figures for IgE level. Results of individual specific IgE level among those patients who tested positive was as follows; house dust mite $61.7 \%$, grass pollens $54.2 \%$, dairy/cow's milk $39.9 \%$, nuts $16.7 \%$, dog dander $15.9 \%$, cat epithelium $13.6 \%$, egg $9.7 \%$, wheat $4.9 \%$ and fish/seafood $3.7 \%$. On follow up, a significant number of patients reported and improvement in their atopic dermatitis symptoms after introduction of allergen avoidance strategy.

Conclusion: RAST testing is a valuable tool in investigating and managing atopic dermatitis.

\section{Introduction}

Atopic dermatitis is a chronic itchy inflammatory skin condition which can affect up to $2 \%$ of the population [1]. It has hereditary predisposition affecting infants, children extending to adulthood. In children different studies demonstrated prevalence of $8-20 \%$ $[1,2]$. In children a strong association has been demonstrated between IgE mediated food allergy and atopic dermatitis [3]. Atopic dermatitis is usually linked to certain allergies [3] and despite the term atopic, up to $60 \%$ of children with the condition do not demonstrate IgE- mediated sensitivity to allergens [4]. In general patients suffer from atopic dermatitis or parents of young patients often suspect allergies causing the problems and they point out food as a cause or flare up factor. However, only a small percentage specifies these as a possible factor [5]. The clinical picture and presentation can also be more complicated by other factors including psycho-emotional, environmental, viral or bacterial infection [5]. Atopic dermatitis was viewed to be an allergic disease for a period [6,7]. Immune mediators in maternal breast milk influence the development of childhood eczema in that elevated Li1B, IL-17 of CCL17 is associated with more than a halved risk of childhood eczema in a high-risk cohort $[8,9]$. There are many tests advocated to be helpful in the diagnosis, identifying the triggering factor and grading of atopic dermatitis. These include skin prick testing, RAST, Food challenge and Patch Test. Other commercially available tests not necessarily recommended by doctors due to the 
lack of scientific evidence supporting them include allergy testing kits, such as hair analysis tests, kinesiology tests and VEGA tests. In the United Kingdom patients with atopic dermatitis are usually seen by Primary Care General Practitioners. Diagnosis and initial treatment are usually given. Only those who fail to respond sent to the secondary care for specialist assessment and management.

\section{Materials and Methods}

133 patients under the age of 12 were enrolled. Standard RAST testing was requested for all. This included dairy products, house dust mite, grass, nuts, seafood, cereals, dog dander and cat epithelium. The result and lever of IgE $(\mathrm{IU} / \mathrm{ml})$ was graded as follows: -ve if IgE (IU/ml) is less than 0.35, Grade 1: 0.35-0.69, Grade 2: 0.70-3.49, Grade 3: 3.5-17.49, Grade 4: 17.50-52.49, Grade 5: 52.50-99.99, Grade 6>100.52.49.

\section{Results}

One hundred and thirty-three children with eczema were referred to the Royal Wolverhampton NHS Trust were tested. RAST test showed that $91.7 \%$ of children (122/133 patients) having high/abnormal figures for IgE level (Figure 1). Results of individual specific IgE level among those patients who tested positive (Figure 2) was as follows; house dust mite $62.7 \%$, grass pollens $54.2 \%$, dairy/cow's milk 39.9\%, nuts $16.7 \%$, dog dander $15.9 \%$, cat epithelium $13.6 \%$, egg $9.7 \%$, wheat $4.9 \%$ and fish/seafood $3.7 \%$, wheat $1.2 \%$ and soya $0.9 \%$. As for the severity level of IgE measured for the scoring levels above 52.50 (IU/ml), Grade 5 and 6 (Figure 3), house dust mite (49.2\%), nuts (11.5\%), dairy products/egg (9.7\%) and grass (54.2\%). In the moderate grading of severity (3.5-52.49 IU/ml) Grade 3 and 4 dairy products/egg (9.7\%), nuts (9.7\%), house dust mite (18.1\%), cat epithelium (14.7\%), dog dander (14.4\%), wheat (3.1\%) and soya all (1\%). On follow up, a significant number of patients reported an improvement in their atopic dermatitis symptoms after introduction of allergen avoidance strategy.

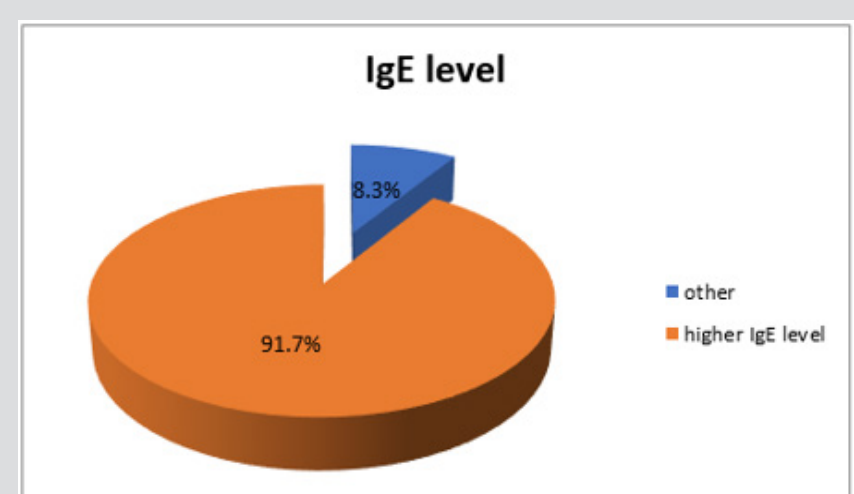

Figure 1: Percentage of patients with atopic dermatitis showing high IgE level which is 122 patients out of the 133 who participated in this study.

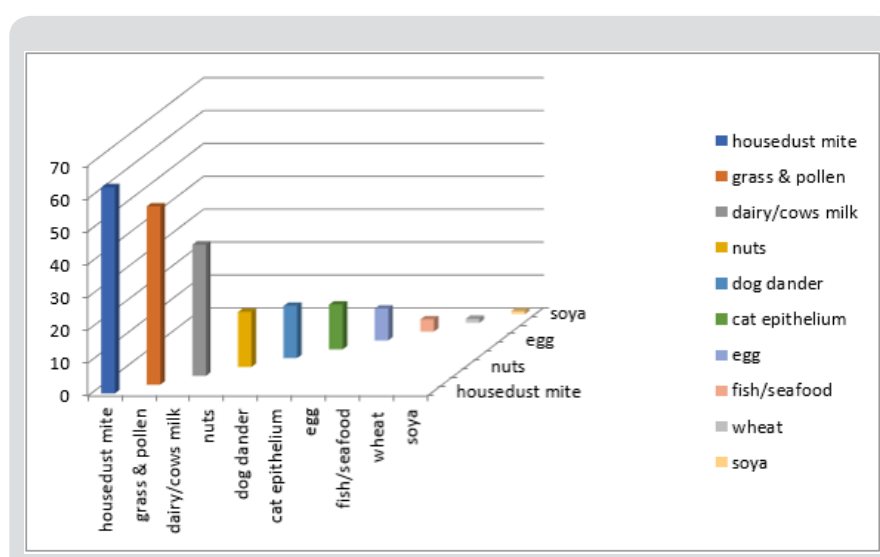

Figure 2: This figure demonstrates the individual specific IgE levels among patients who tested positive for RAST testing.

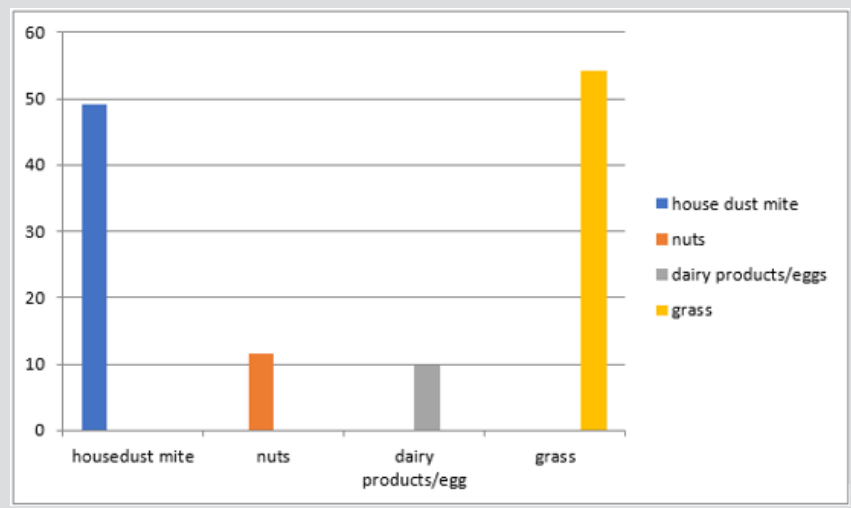

Figure 3: This figure demonstrates the grade $5 \& 6$ severity positive RAST tests in the patients who were studied.

\section{Discussion}

The relationship of atopy, dermatitis and IgE mediated food allergy is not clear; neither extensive research has been conducted in the field. IgE mediated food allergy and atopic dermatitis prevalence peak at the first 12 months of life. The risk of atopic dermatitis in infancy increase by threefold with high levels of IgE sensitization to foods. Children with atopic dermatitis have higher prevalence of food allergy. IgE mediated food allergy affects $35 \%$ of children with moderate to severe atopic dermatitis and 6-8\% of asthmatic children have wheezing which is thought to be food induced although $80 \%$ of those outgrow their food allergy [9]. In a recent study $27 \%$ of patients diagnosed with atopic dermatitis suffer from food allergy to wheat, soya, egg, peanuts and milk, while $32 \%$ suffer from sensitisation to these foods. Also, it found that food allergy, can act as a triggering factor in provoking and flare up of atopic dermatitis children in 7\% [10]. Our study was aimed to evaluate the importance of carrying out RAST testing in patients with atopic dermatitis referred to the specialist secondary care hospital within the National Health Service in the UK. These 
patients usually moderate to severe grade as most of the mild cases are treated by General Practitioners in primary care. We found high population of these patients to have high total serum IgE levels at $91.7 \%$. High severity grades of allergens specific IgE were also detected in these patients and this helped to guide patients to which specific allergen to avoid.

Despite the wealth of information in support of the link between IgE mediated allergy, RAST testing and atopic dermatitis a study showed that some patients with positive tests have no clinical manifestations of disease when exposed to the allergen [11]. Atopic dermatitis or eczema often occurs first in the atopic march or atopic triad of eczema, asthma and hay fever [12]. A positive for an allergen specific IgE measured by RAST indicates a sensitised state to the allergen which is not synonymous to a clinical diagnosis. The RAST results provided by our study helped to increase our ability to rank allergens according to their likely contribution to the symptoms of each patient. The significant reporting by atopic dermatitis patients in improvement of symptoms after avoiding the possible offending allergen gives promising evidence that specific allergen avoidance, guided by RAST, can contribute positivity in the management of atopic dermatitis. However, there still need to be further investigations and assessment to draw a conclusion.

\section{Conclusion}

RAST testing is an available tool in investigating and managing of atopic dermatitis. It has been undervalued and underestimated in the past. Our results demonstrated significant positive results to an association of severity of atopic dermatitis and the grade of allergen specific IgE antibody. Therefore, we recommend considering RAST testing to all those children with atopic dermatitis who failed first line of treatment.

\section{ISSN: 2574-1241}

DOI: 10.26717/BJSTR.2019.18.003108

Mohammed Al-Abadie. Biomed J Sci \& Tech Res

This work is licensed under Creative Commons Attribution 4.0 License

Submission Link: https://biomedres.us/submit-manuscript.php

\section{References}

1. Kay J, Gawkrodger DJ, Mortimer MJ, Jaron AG (1994) The Prevalence of Childhood Atopic Eczema in a General Population. J Am Acad Dermatol 30(1): 35-39.

2. Eichenfield LF, Ellis CN, Mancini AJ, Paller AS, Simpson EL (2012) Atopic dermatitis epidemiology and pathogenesis update. Semin Cutan Med Surg 31(3 suppl): 53-55.

3. Hill DJ, Hosking CS (2004) Food Allergy and atopic dermatitis in infancy: an epidemiologic study. Paediatric Allergy and Immunology 15(5): 421427.

4. Flohr C, Johansson SGO, Vahlgren CF, Williams HC (2004) How Atopic is Atopic Dermatitis? J Allergy Clin Immunol 1149(1): 150-158.

5. William HC (2005) Clinical Practice: Atopic dermatitis. N Eng/J Medicine 352(22): 2314-2324.

6. Halbert AR, Weston WL, Morelli JG (1995) Atopic dermatitis: is it an allergic disease. J Am Acad Dermatol 33(6): 1008-1018.

7. Zeiger R, Heller S (1989) Effect of combined maternal and infant food-allergy avoidance on development of atopy in early infancy: A randomised study; J ALLERGY CLIN IMMUNOL 84(1): 72-89.

8. Hill DJ, Sporik RS, Thorburn J, Hesking CS (2000) Association of atopic dermatitis in infancy with immunoglobulin E food sensitisation. J Pediatric 137(4): 475-479.

9. Jepren AA, Clewes BL, Carson CG, Schoos AM, Thysen AH, et al. (2016) High breast milk IL is associated with reduced risk of childhood eczema. Clinical Allergy Journal 46(10): 1344-1354.

10. Celakovska J, Ettlerova K, Ettler F, Vaneckova J, Butac J (2015) Food and Agricultural Immunology 26(1): 26-37.

11. Cox L, Williams B, Sicherer S (2008) American College of Allergy, Asthma and Immunology Test Task Force; American Academy of Allergy, Asthma and Immunology Specific IgE Test Task Force. Pearls and pitfalls of allergy diagnostic testing: report from the American College of Allergy, Asthma and Immunology/American Academy of Allergy, Asthma and Immunology Specific IgE Test Task Force. Ann Allergy Asthma Immunol 101(6): 580-592

12. Jingying Wang, Alban Ramette, Jurca M, Goutaki M, Breadsmore CS, et al. (2017) Association between breastfeeding and Eczema during childhood and adolescence: A cohort study 12(9).

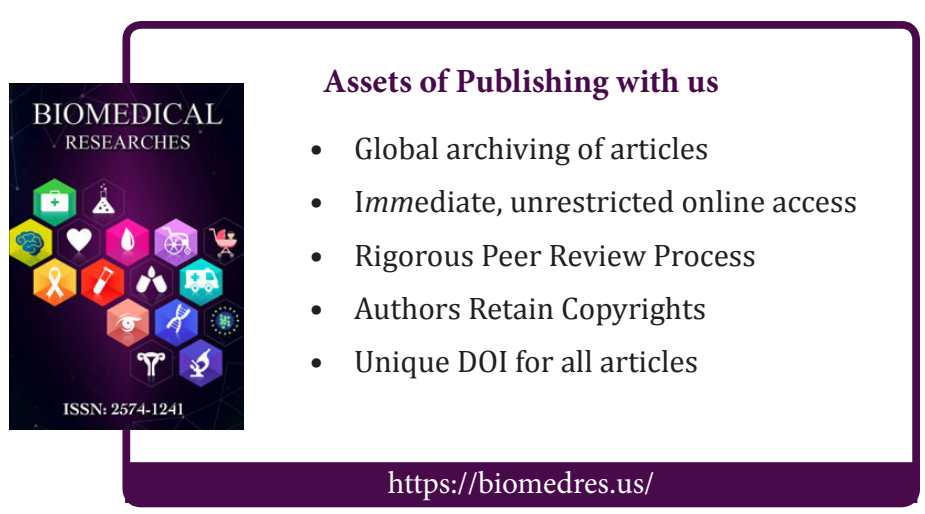

